\title{
BMJ Open Improving the quality of child-care centres through supportive assessment and 'communities of practice' in informal settlements in Nairobi: protocol of a feasibility study
}

\author{
Margaret Nampijja (D) ,' Kenneth Okelo, ${ }^{1}$ Patricia Kitsao Wekulo, ${ }^{1}$ \\ Elizabeth W Kimani-Murage, ${ }^{1}$ Helen Elsey ${ }^{2}$
}

To cite: Nampijja M, Okelo K, Wekulo PK, et al. Improving the quality of child-care centres through supportive assessment and 'communities of practice' in informal settlements in Nairobi: protocol of a feasibility study. BMJ Open 2021;11:e042544. doi:10.1136/ bmjopen-2020-042544

- Prepublication history for this paper is available online. To view these files, please visit the journal online (http://dx.doi. org/10.1136/bmjopen-2020042544).

Received 07 July 2020

Revised 19 December 2020

Accepted 18 January 2021
Check for updates

(c) Author(s) (or their employer(s)) 2021. Re-use permitted under CC BY-NC. No commercial re-use. See rights and permissions. Published by BMJ.

${ }^{1}$ Maternal and Child Wellbeing Unit, African Population and Health Research Center, Nairobi, Kenya

${ }^{2}$ Department of Health Sciences, University of York, York, UK

Correspondence to

Dr Margaret Nampijja;

maggie.nampijja@gmail.com

\section{ABSTRACT}

Introduction Investing in children during the critical period between birth and age 5 years can have longlasting benefits throughout their life. Children in Kenya's urban informal settlements, face significant challenges to healthy development, particularly when their families need to earn a daily wage and cannot care for them during the day. In response, informal and poor quality child-care centres with untrained caregivers have proliferated. We aim to co-design and test the feasibility of a supportive assessment and skills-building for child-care centre providers.

Methods and analysis A sequential mixed-methods approach will be used. We will map and profile childcare centres in two informal settlements in Nairobi, and complete a brief quality assessment of 50 childcare centres. We will test the feasibility of a supportive assessment skills-building system on 40 child-care centres, beginning with assessing centre-caregivers' knowledge and skills in these centres. This will inform the subsequent co-design process and provide baseline data. Following a policy review, we will use experience-based co-design to develop the supportive assessment process. This will include qualitative interviews with policymakers $(n=15)$, focus groups with parents ( $n=4$ focus group discussions (FGDs)), child-care providers ( $n=4$ FGDs) and joint workshops. To assess feasibility and acceptability, we will observe, record and cost implementation for 6 months. The knowledge/skills questionnaire will be repeated at the end of implementation and results will inform the purposive selection of 10 child-care providers and parents for qualitative interviews. Descriptive statistics and thematic framework approach will respectively be used to analyse quantitative and qualitative data and identify drivers of feasibility.

Ethics and dissemination The study has been approved by Amref Health Africa's Ethics and Scientific Review Committee (Ref: P7802020 on $20^{\text {th }}$ April 2020) and the University of York (Ref: HSRGC $20^{\text {th }}$ March 2020). Findings will be published and continual engagement with decisionmakers will embed findings into child-care policy and practice.

\section{Strengths and limitations of this study}

- Our close engagement with child-care providers through experience-based co-design and the use of communities of practice is likely to enhance the acceptability and sustainability of the supportive assessment system.

- The mixed methods design involving various stakeholders in child-care provision will enable triangulation of data and enhance the richness of our findings

- The phased approach will allow for ongoing modification of the model based on the lessons learnt.

- Anonymised geo-mapping of child-care centres in relation to health and other essential services is added value in linking these centres to the services.

- The study focusses on the feasibility of the intervention; future studies are needed to test costeffectiveness of the intervention in improving the quality of child-care centres and child health and development.

\section{INTRODUCTION}

There is clear evidence that investing in early childhood development (ECD) during the critical period between birth and 5 years of life can have long-lasting benefits in the life of the child, ${ }^{1}$ reduce health inequities and boost individual, social and economic development. ${ }^{1-5}$ Increasing global focus on early childhood health and development is enshrined within the Sustainable Development Goals (SDGs), particularly SDG 4 which includes targets relevant to young children's health, safety and development. ${ }^{6}$ Further, global leadership comes from the $71^{\text {st }}$ World Health Assembly where, in 2018 the Nurturing Care Framework for Early Childhood Development ${ }^{7}$ was established to provide a broad framework for supporting the development of children from pregnancy 
up to age 3. Despite this, 250 million children aged less than 5 years are at risk of not achieving their full developmental potential, ${ }^{1}$ the majority $(67 \%)$ of whom are from sub-Saharan Africa (SSA). Multiple adverse exposures including poverty, malnutrition, disease, exposure to injuries and unstimulating environments underlie suboptimal child development in low- and middle-income countries (LMICs) and children living in extremely impoverished settings are particularly at risk since poverty limits access to quality healthcare, balanced diet, quality education and a nurturing home or preschool environment. ${ }^{28}$

Despite the increased focus on ECD, limited attention has been given to the development and provision of childcare centres in LMICs. The focus on child-care is particularly important in this era of rapid urbanisation, with over half the world's population living in urban settings. It is estimated that by $2050,56 \%$ of the population in Africa and $64 \%$ in Asia will be living in towns and cities. ${ }^{9}$ Urbanisation brings with it social, economic and cultural changes and has been identified, in itself, as a determinant of health. ${ }^{10}$ Increased urbanisation has brought changing working patterns with increases in female employment outside the home, resulting in a pressing need for childcare options particularly in low-income urban settings. Quality child-care centres have the potential to provide multiple benefits to children, families and societies ${ }^{7}$ through women's participation in the labour force. ${ }^{11-15}$ A framework for providing quality child-care is found in WHO's Nurturing Care guidelines. The framework specifies an environment that is healthy, safe, hygienic, enables nutritious food and provides stimulating nurturing care. ${ }^{7}$ Ensuring centre care-givers understand these elements and can apply them to their centres is a priority for programmes that train and support centre-based care providers. Increases in parental employment, particularly of mothers, has the potential to provide indirect benefits to the child through increased household income and improved nutrition. ${ }^{16}$ Evidence for the impacts of childcare on children's cognitive, socio-emotional and physical health is more limited both in high-income and LMIC contexts. ${ }^{1617}$

The role of a stimulating environment is increasingly becoming recognised and emphasised..$^{18} \mathrm{~A}$ well-facilitated child-care centre that provides opportunities for learning and play, good feeding and access to healthcare has the potential to nurture and optimise child development. ${ }^{19-22}$ On the other hand, child-care centres with limited cognitive stimulation are likely to hold back children's development. ${ }^{23}$

The Government of Kenya has outlined guidelines for child-care centre such as the Early Childhood Development Service standard guidelines that were instituted in 2006. ${ }^{24}$ However, due to lack of resources, limited training, low supervision and absence of assessment tools, many child-care centres do not meet the minimum standards of care ${ }^{25}$ for example, the Kenya ECD guidelines. ${ }^{24}$ This situation is particularly acute in informal settlements where providers, who are almost exclusively women, are frequently untrained and unsupported and offering care in one or two rooms with limited facilities to provide a hygienic, safe and stimulating environment. Estimates of the number of such child-care centres in informal settlements in Nairobi put the figure at $2700 .^{25}$ Therefore, many children are at risk of receiving inadequate care and nurturing during the critical period of their development, which in turn negatively impacts on their future learning and well-being.

We aim to co-design and test the feasibility of a system for supportive assessment and skills building of child-care centre providers, with the long-term vision of improving the quality of paid child-care for children in poor-urban settings. The quality of child-care centres in LMICs particularly in impoverished settings is poor due to lack of resources, lack of skills on the part of the caregivers and absence of clear guidelines to regulate centre-based child care. Hence the focus of the present work is to improve the quality of centre-based child care in line with the WHO's nurturing care guidelines, through provision of education and supportive supervision by local community workers or volunteers. We also aim to review national and county policies relevant to early years care to identify gaps that will inform efforts for improvement. The co-design approach will allow identification of an appropriate cadre of local community workers or volunteers who are then trained to assess the quality of the child-care centres with a particular focus on safety, health and nurturing care using a simple assessment tool. This will inform the skills building for the care providers to enable them improve the quality of care they provide. Our long-term vision is expressed in our Theory of Change in figure 1.

\section{METHODS AND ANALYSIS}

\section{Study design}

We will use a sequential mixed-methods ${ }^{26}$ design comprising three phases (illustrated in figure 2) to answer the following objectives:

1. To map and assess the child-care environment and provider skills in informal settlements;

2. To co-design with child-care providers, parents, government and ECD experts a supportive assessment and skills-building community of practice $(\mathrm{CoP})$ approach which can be delivered at scale within informal settlements in Kenya;

3. To assess the feasibility and costs of delivering the codesigned model over a 6 months' period in two informal settlements in Nairobi: Korogocho and Viwandani.

\section{Setting}

The study will be conducted in two informal settlements i.e. Korogocho and Viwandani in Nairobi, Kenya. These two settlements have been selected as they are a home to large numbers of women working outside the home, frequently in informal employment. To meet the childcare needs of these families, there are many informal (low quality) but affordable child-care centres. The 

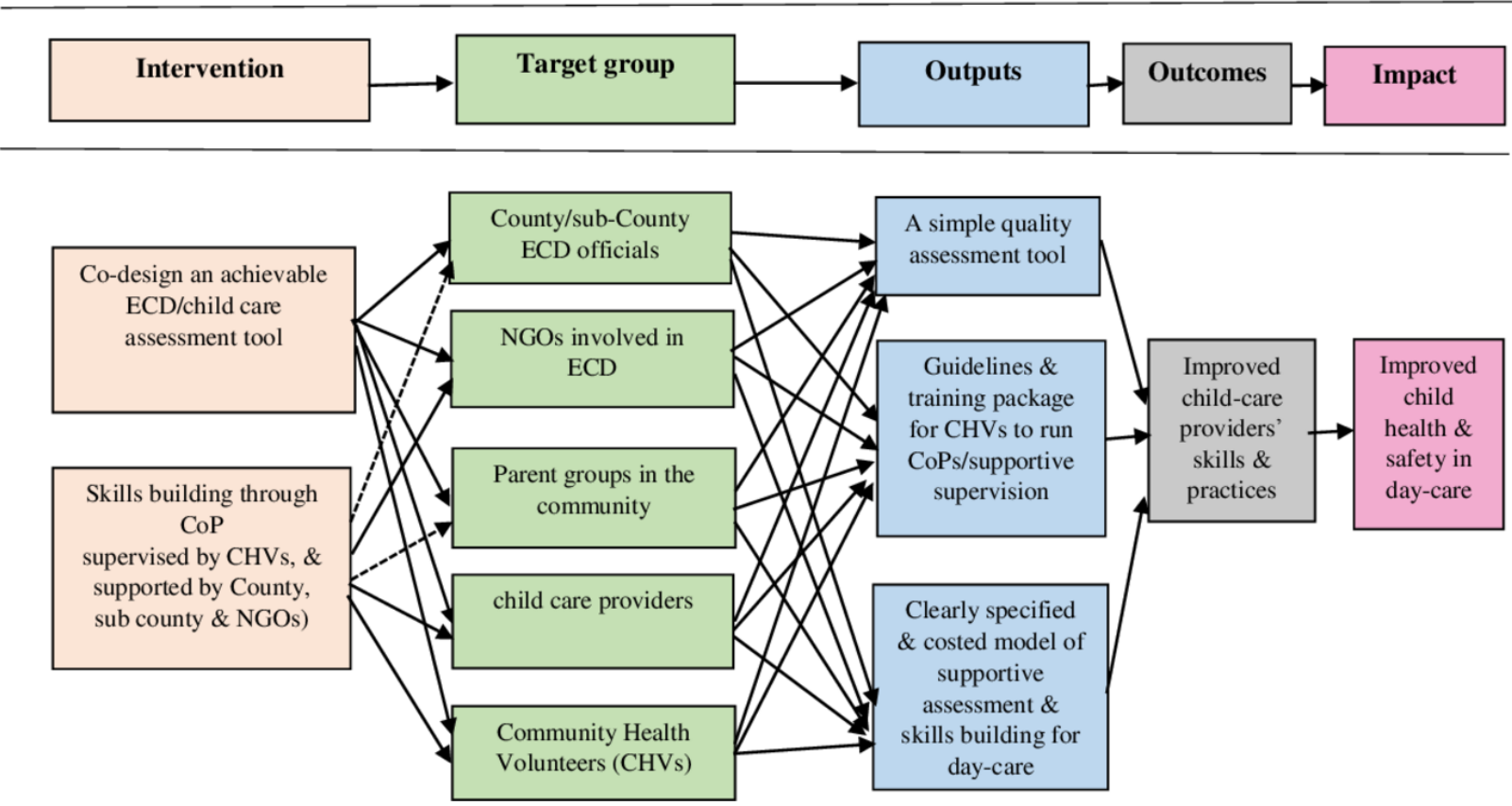

Figure 1 Theory of change showing long-term vision for the intervention. This illustrates how supportive assessment and skills building co-designed together with various stakeholders is expected to lead to improvements in the skills and practices of the child care providers and ultimately result into improved child health and safety in the day care.

socio-demographics of these communities have been well characterised by the Nairobi Urban Health and Demographic Surveillance System, ${ }^{27}$ within the African Population and Health Research Center (APHRC). APHRC is the lead research organisation for this feasibility study. Korogocho and Viwandani, located about $7 \mathrm{~km}$ from each other, are densely populated with 63318 and 52583 inhabitants per square $\mathrm{km}$, respectively. The settlements are characterised by poor housing, poor sanitation, lack of basic infrastructure, insecurity, high crime rate and poor access to maternal and child health (MCH) services and healthcare in general. ${ }^{27}$ The two communities were selected because they represent the poverty spectrum on which informal settlements in Nairobi lie, with Viwandani (which is close to the industrial area) being relatively less poor than Korogocho. This variation will support the transferability of our findings to a wide range of urbanpoor setting in the East-African region.

\section{Procedures}

Phase 1: mapping and assessing the child-care environment in informal settlements

\section{Mapping of child-care centres}

In the first 3 months, we will map all child-care centres in Korogocho and Viwandani informal settlements using OpenStreetMap. Community members from informal settlements will be trained as field interviewers to conduct the mapping. Within their designated villages, field interviewers will visit households asking if there are any centres where staff are paid to look after children under the age of 5 . All the 14 villages in the two settlements will be mapped. Once the trained mappers identify a childcare centre, they will record this using OpenStreetMap.
These data will not be uploaded to the OpenStreetMap site but kept confidentially with the team. This will ensure that child-care providers' privacy is maintained. After taking consent from the centre provider, we will record a minimum data set by asking the provider questions, observing the facilities and practices in the childcare centre and checking any records available. This will allow us to capture basic information such as opening hours, staffing levels, number and age of children, rooms, hygiene facilities, fees, any organisational/nongovernmentalorganisation (NGO) support, name of local community health volunteer (CHV), among others. The child-care centre providers will also be asked if they wish to be involved in more detailed quality and skills assessment and to be part of the supportive assessment model. Contact details of those willing to participate further will be recorded. Existing data held within APHRC on the location of health facilities within Korogocho and Viwandani will enable us explore the distribution of health facilities in relation to the child-care centres within the area. During the co-design process this will enable us to see which health facilities are most appropriate for providers to seek healthcare for the children in case of an emergency.

\section{Assessing the child-care environment}

Prior to the co-design phase, we will develop a simple quality assessment tool drawing on tools currently used by an NGO, Kidogo, who run child-care centres in informal settlements in Nairobi and are partners on this study. Tools such as the Family Child Care Environment Rating Scale-Revised ${ }^{28}$ have been considered for use; however, as many such tools have been developed and used in 


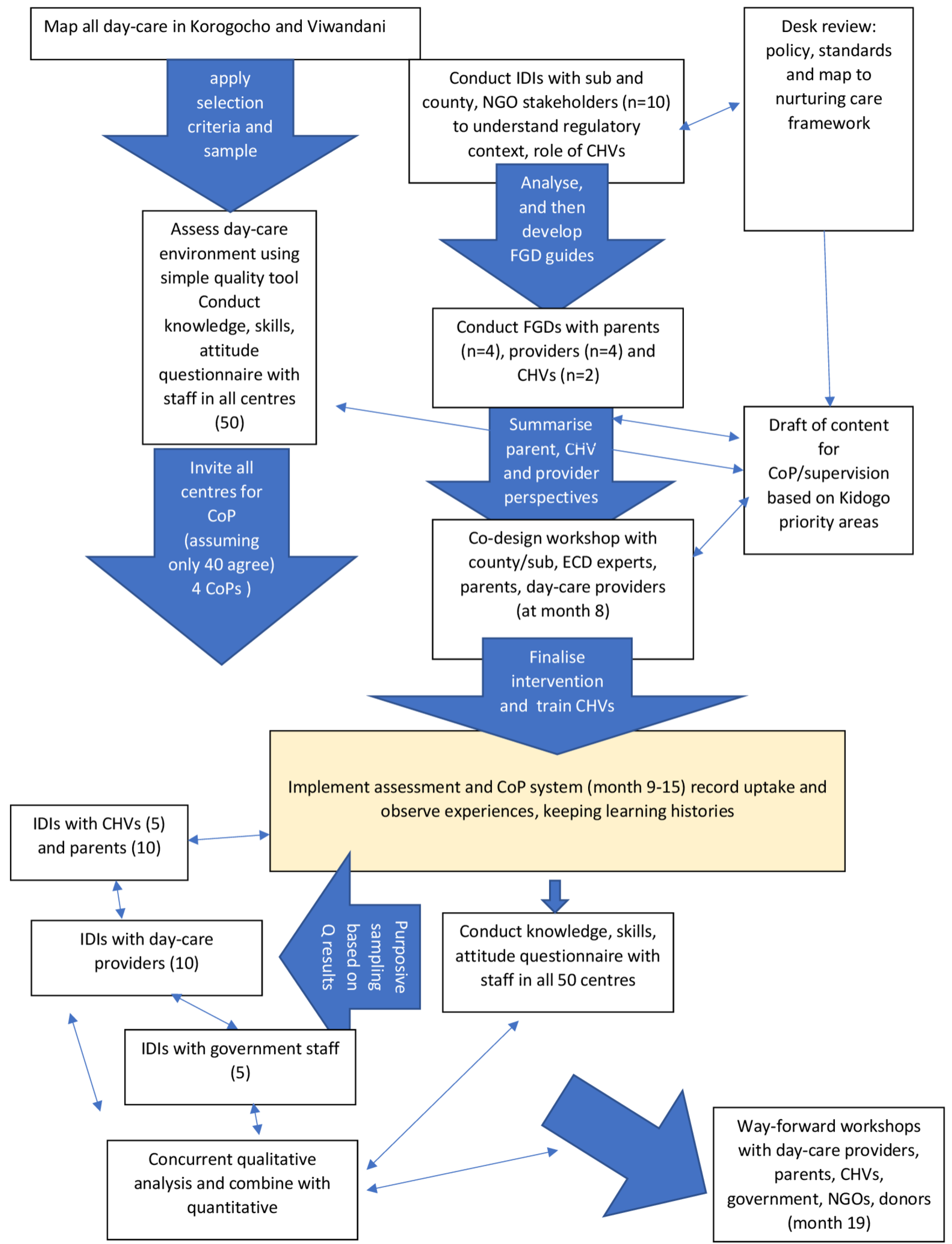

Figure 2 The flow of data collection and co-design activities. This shows the sequence of how the different data collection and co-design activities will be conducted. Activities that come earlier (on top) inform subsequent activities and so on. CoP, community of practice; CHV, community health volunteer; ECD, early childhood development; FGD, focus group discussion; IDI, in-depth interviews; NGO, non-governmental organisation. 
high-income contexts, items require considerable adaptation to the context of informal settlements. Further, we plan to design the tool so that it can be used within routine practice by a CHV or other community worker, to support the improvement of the centres. We anticipate that the simple quality assessment tool will be revised during the co-design and implementation process as we learn more about its feasibility, appropriateness and the kind of information required.

\section{Assessing child-care centre staff knowledge, skills and attitudes}

Questionnaires will be administered to the child-care providers to assess their knowledge, skills, attitudes and opportunities/barriers to implement this knowledge and attitudes within the areas of stimulation, nutrition, health and safety, staff and training, parent involvement and resource management. This questionnaire will inform the content of the CoP sessions and the allocation of childcare centre providers to specific CoPs to enable them to share different skills through peer learning.

Currently, the number of child-care centres in Korogocho and Viwandani is not known. The numbers may therefore be too large for all to be assessed using the simple quality assessment tool. We estimate that our field interviewers can conduct assessments and knowledge, skills and attitudes within 50 centres. This number has been agreed on based on the time and resources available to the project. No formal sample size calculation has been done. However, this figure will provide sufficient insights into the provision of child-care services. If the number of child-care centres in Korogocho and Viwandani exceeds 50 , we will randomly sample centres proportionate to the total number of centres in each slum to reach a total sample of 50 . We will stratify our random sample to include both centre-based and home-based providers.

Phase 2: co-designing a monitoring and support system for quality improvement of child-care services

This phase will run concurrently with Phase 1 . We will begin with a desk review of the existing national and county-level policies and guidelines relevant to ECD and child-care to understand the extent to which they deal with the challenges of child-care within informal settlements and how they incorporate the key components of the nurturing care framework. ${ }^{7}$ This analysis will inform the co-design process outlined in the following section.

\section{Co-design of a supportive assessment and skills building CoP approach}

We will draw on approaches used in experience-based co-design $(\mathrm{EBCD})^{29}$ to develop a simple process of quality assessment and support. EBCD uses a process of focus groups with users, implementers and decisionmakers and feeds findings from these interactions into a series of co-design workshops to ensure that voices of all actors are heard. We will begin with a series of focus group discussions (FGDs) with parents (four FGDs: two in Viwandani and two in Korogocho), and child-care centre providers (four FGDs: two in Viwandani and two in Korogocho). The FGDs with parents will explore their current child-care use and experiences and their preferences, including willingness to pay for different models and standards of child-care. We will purposively select mothers and fathers of different ages, varying numbers of children in the household and different occupations, as identified by the child-care providers. This will enable a range of perspectives and child-care needs to emerge. The FGDs with child-care providers will explore needs for support and their perceptions of the potential supportive assessment model. We aim to use the CoP model, which, drawing on the situated learning theory ${ }^{30}$ allows peers to share their experiences and practice-based knowledge to learn and improve their practice, rather than through formal instruction or training. The model has been successfully used to harmonise ECD services among various stakeholders in South Africa. ${ }^{31}$ The ideas that emerge from various discussions will be shared with childcare providers to enable them to shape the nature and modalities of the CoP model.

In parallel with the mapping and co-design work, we will conduct a qualitative review of national and county policies relevant to early-years care. This review and the findings from the qualitative methods with providers and parents will inform interview guides to be used within in-depth interviews $(n=5)$ with county, subcounty officials and NGO representatives. These interviews will inform decisions on the most suitable cadre to deliver the supportive supervision and CoPs in informal settlements. We envisage that these will be the existing CHVs; however, this will be agreed on during the co-design process. Once identified, we will conduct two FGDs (one in Viwandani and one in Korogocho) with CHVs or other identified cadre, to understand their motivations and suitability for the new role and how this would fit within their existing workload. Discussions will be held with their supervisors (community health assistants) and managers at subcounty and county level. Throughout the process, we will check back with government officials to clarify any issues raised and obtain their feedback on emerging issues.

A co-design workshop will be held bringing together parents, child-care centre providers, government staff and child-care/ECD experts. Findings from the FGDs and the quantitative assessments of the centres will be presented to ensure that the development of the supportive supervision and CoP model is grounded in the realities of childcare in informal settlements.

Materials to be used to provide information and support to the child-care providers through the CoPs and supportive assessment process will be developed based on those already used and successfully evaluated by our partner Kidogo to support and train centre caregivers. The content of each CoP session will focus on the areas of weakness identified in the assessments and those that emerge through discussions with caregivers. We envisage that during the 6-month implementation, key areas of focus will include child health, nutrition, hygiene and best 
ECD practices. Areas such as management and finances may also be included depending on identified needs.

The output of the EBCD process will be a clearly specified supportive assessment and skills-building process, including the use of a simple quality assessment tool, and agreement on the implementation modalities of the supportive supervision system and the content of the CoPs.

The 50 centres assessed at base-line will be invited to participate in a 6-month pilot of supportive supervision and CoP sessions. Based on Kidogo's experience of supporting child-care providers through CoPs in other slums, we assume that $20 \%$ may not wish to be involved. With 40 interested child-care providers, we estimate that four CoP groups (two in Viwandani and two in Korogocho) with additional supportive assessment visits from the CHVs could be conducted. Within the CoP groups, child-care centres will be paired/matched to support each other based on their strength and weakness identified in the knowledge, skills and attitudes questionnaires and centre assessments and also on their geographical location.

The CHVs (or similar cadres) will conduct centre visits according to a schedule agreed on during the co-design process. They will use the simple assessment tool to monitor any changes in the child-care environment including care providers' skills and practices, and advise as necessary. These data will be included in our evaluation.

To encourage participation, centres will receive a certificate of participation in the project, and care-providers that were involved in the $\mathrm{CoP}$ intervention will receive a certificate for having undergone a participatory training in child-care. These incentives will be organised through the Nairobi City County Education Department.

We will record the process of co-design and analyse key sticking points and enablers. This will contribute to answering objective 3 . We will use the 'learning histories' method ${ }^{32}$ to capture and record key events chronologically, and ask key stakeholders for their views on the barriers, facilitators and experiences with the different aspects/components of the CoP as well as the guidelines.

Phase 3: assessing the feasibility, acceptability and costs of delivering the co-designed model over a 6-month period in two informal settlements in Nairobi - Korogocho and Viwandani

We will analyse the results of the quality assessments conducted by CHVs and document and cost all aspects of the supportive assessment and CoP intervention which will include:

- The training and supervision of CHVs (or similar) who deliver the intervention;

- The number of supportive supervision visits made by each CHV (or similar) to child-care centres;

- The number of CoP groups established and sessions run, a record of participants in each group (number and names), the duration and topics covered. This will be recorded by the CoP facilitator, that is, $\mathrm{CHV}$ or similar.

To gain a more in-depth assessment of the feasibility and acceptability, and content of the supportive supervision and CoP sessions, the research team will conduct observations on a proportion of the CoP sessions and supportive assessment visits. We estimate that we will be able to observe eight CoP sessions (ie, two for each of the four CoP groups) and 10 child-care centre visits over the 6-month implementation period. These observations will follow an observation guide which will record topics covered, any facilitators and barriers facing the child-care providers in implementing the recommended skills and practices and reflections on the interaction between the CHVs (or similar) and child-care providers.

After 6months of implementation, we will evaluate the extent to which the supportive assessment and CoP approach is able to build care-giver skills in the six main areas through a follow-up of the knowledge and attitudes questionnaire delivered in Phase 1 to all child-care staff involved in the CoP. We will also conduct an end line assessment of the quality of the child-care environment in each centre to determine improvement since baseline. In line with our sequential mixed methods approach, the results of the questionnaires and any change in scores will inform the sampling of approximately 10 child-care providers for qualitative interviews to understand experiences from both those who show particular improvements and those who are not able to improve or decide not to engage in the intervention. The interviews will highlight the acceptability of the intervention and any challenges and barriers they face in improving quality in their child-care centres.

In addition, we will analyse the monitoring data collected by CHVs (or similar) using the simple quality assessment tool to assess their use of the tool as well as any changes in child-care practices. Issues arising through this process will be recorded in the learning histories document.

In line with our sequential design, any variation in the collection of this data and the observation of CHVs practice during the 6 months' implementation will also influence our purposive selection for qualitative interviews (approximately five) with the CHVs (or similar) and the issues to be covered in the interview guides. The interviews will enable the identification of the barriers and facilitators to the implementation (including acceptability) of the supportive supervision and CoP system in the child-care centres in the two slums.

We will also conduct qualitative interviews (approximately 10) with parents (both mothers, fathers and any other primary care-givers) whose children use the childcare centres who have been involved in the CoPs to understand if they have noticed any changes or have any feedback on the supportive assessment and CoP model. We will also interview (approximately five) government staff (subcounty and county level) to identify any facilitators and barriers to implementation and their views on possibilities for scale-up. Again, in line with the sequential design, we will draw on the analysis of qualitative methods and the assessments conducted in the previous phases to inform the interview guides used.

Assessment of acceptability will be done together with feasibility assessment within the qualitative interviews 
particularly at the end of the intervention. Records and observations during implementation will highlight uptake of the CoP sessions and numbers of supportive assessment visits. Qualitative interviews with CHVs will focus on their attitude towards delivering the supervision and supporting the monthly sessions. Interviews with centre care providers will focus on their experiences and perceptions of the intervention, and how positive they feel to embrace the support provided. We will compare the findings from these different data sources, both quantitative and qualitative to understand acceptability of the intervention.

We will identify all costs associated with the delivery of the $\mathrm{CoP}$ and supervision system and compare with the benefits gained in terms of care-giver skill and child-care centre improvements. We will identify the costs of implementation of the assessment of standards/guidelines including CHV time to conduct assessments, and county supervision input. The information will be fedback to and discussed with county and national level decision makers in a final workshop.

Figure 2 illustrates the flow of data collection and co-design activities.

\section{Data analysis}

Descriptive statistics (frequencies, means, medians, SDs and IQRs) will be used to describe the data from the quality assessment tool and the knowledge and attitudes questionnaires at baseline. Data from each quantitatively measured outcome will be summarised using proportions for binary outcomes and means or medians for continuous outcomes.

Crude comparisons of changes in the baseline and end line quality assessment tool and the knowledge and attitudes questionnaires will be done using simple hypothesis tests that is, t-test and $\chi^{2}$ for continuous and categorical outcomes, respectively. The primary focus of this project is to test the feasibility of the co-designed CoP model and indicators of improvements in the quality, and the knowledge and attitude of the centre care providers. Hence the sample size has not been powered to support complex data analyses such as modelling and adjusting for multiple confounding factors.

Qualitative data from the interviews, focus groups, observations and learning histories will be analysed using a thematic framework approach. ${ }^{33}$ The framework will include themes on feasibility, acceptability and experiences of the supportive assessment and skills building system. The policy review and policy-related interview data will be analysed using the policy analysis framework developed by Walt and Gilson, ${ }^{34}$ which distinguishes actors (individuals, organisation or the state and their actions that affect health policy), context (political, economic, social both national and international which may affect policy), content (substance of the policy) and process (the way policies are developed and implemented). This framework has been chosen as it is amenable to use in policy analysis in LMIC contexts and provides sufficiently broad constructs to facilitate analysis. Our mixed methods approach will draw meta-inferences from both our quantitative results and qualitative findings from all phases of the study. ${ }^{26}$ The meta-inferences will be devised and discussed across our full team and will be presented back to those involved in the supportive assessment and CoP model throughout the study in a final dissemination workshop to ensure that our interpretation of the data adequately reflects their perspectives.

\section{Patient and public involvement}

As a community-based study, there is no involvement of patients. However, early in the development of the protocol, we consulted the County government and a few other stakeholders on the state of child care in the County and what they thought were major gaps and how we can work together to address some of the gaps using a community of practice approach. While there was not local community involvement in the design of the project, the APHRC team have a long-standing involvement in both informal settlements. The challenges of providing sufficient, quality child-care for the large number of families, particularly women, who work long hours outside the home has been raised persistently by community members. Engagement of parents, community health volunteers and child-care providers throughout the study period is key to an appropriately designed intervention that can be sustainably delivered. In light of this, our project will facilitate active involvement of these groups throughout the co-design process and workshop. The final workshop will provide an opportunity to feedback the lessons learnt from implementation and provide an opportunity for all stakeholders to influence the future implementation, policy and research agenda.

\section{Project timelines}

The project will be conducted over a period of 24 months (November 2019 to October 2021): 5 months for protocol development and ethics; 3 months for community sensitisation and engagement with the various stakeholders; 3 months for mapping/profiling child-care centres including pre-test; 2 months for developing the intervention; 6 months for implementing the intervention; and 4 months for post-intervention assessments and dissemination of findings.

\section{DISCUSSION}

Urban-poor families face significant challenges in providing a safe, nurturing and healthy environment for their children under the age of 5. Within the context of rapid urbanisation, women must work outside the home frequently for long-hours and in unstable informal jobs. They no longer have the support of the extended family that they may have relied on in rural areas to provide care for their children. With an estimated $89 \%$ to $95 \%$ of women working in the informal sector in SSA, ${ }^{35}$ there is an urgent need for child-care solutions for these women. ${ }^{36}$

While formal, well-equipped centre-based care with appropriate facilities and adequate numbers of 
ECD-trained staff may be seen as the ideal, without major investment and subsidisation, such provision within informal settlements is unlikely, particularly in the short-term. There is increasing recognition of the dynamism and entrepreneurial spirit found within informal settlements, challenging a rethink of simplistic dichotomies where the 'informal' is seen as wholly negative and in contrast to formal services. ${ }^{37}$ Supporting and enhancing these existing communitybased informal child-care centres offers opportunities for not only improving child-health, ECD and the livelihoods of working parents, but also building economic opportunities for child-care providers and potentially strengthening social capital within the often transient informal settlements.

Our study will provide valuable insights into how to design and deliver a programme to support informal childcare providers to improve their practices and the quality of the care they provide within the challenging environment of informal settlements. Our continual engagement with local government, policymakers and practitioners will help to explore options for further development of the model and designing follow-on studies to test effectiveness in improving child outcomes (ECD and health), women's empowerment through increased access to employment and sustainability of delivery. Few studies have explored feasible delivery of models of centre-based child-care within informal settlements, yet the need and demand for quality care is great and growing in Nairobi and other LMIC cities. This study should provide useful insights to those in research and practice who are keen to identify effective and appropriate models for increasing access to and improving the quality of child-care in resource-poor settings.

The purpose of this paper is to share information on the process that will be used in co-designing an assessment tool and guidelines for supporting the improvement of the quality of informal child-care centres through a CoP approach. These methodologies together with the findings from the study will be useful for researchers, policymakers, NGOs and other stakeholders working to raise the standards of child-care and ECD particularly in urban-poor contexts.

Overall, the key outputs expected from this study include (1) a simple quality assessment tool in line with the regulations that can be used by CHVs or similar cadres to assess and monitor child-care in urban poor neighbourhoods, and (2) guidelines for supporting childcare centres to improve quality in line with the specified regulations. These are important steps towards improving the health and well-being of young children in urban lowincome settings of Nairobi.

In as much as impact evaluation is important for the intervention, the study is powered to provide an indication of the impact on the care providers' practices but not typically designed to measure its benefits on the quality of child-care, and child health and development. Future studies building on findings from this study, and using sufficient sample sizes, will be useful in determining these effects.

\section{ETHICS AND DISSEMINATION}

The study has received ethical approval from Amref Health Africa's Ethics and Scientific Review Committee ESRC, Kenya (Ref: P7802020 on $20^{\text {th }}$ April 2020) and from the University of York (Ref: HSRGC $20^{\text {th }}$ March 2020). Findings will be published and continual engagement with decision-makers at the county and national level, will embed findings into policy and practice on child-care and ECD.

\section{Dissemination of findings}

Through our previous and ongoing studies, we have established strong links with partners in early childhood development and child-care in the country. We will set up a strategic advisory team to which some of these stakeholders will be included for their input. Engagements with the key stakeholders, including county/subcounty officials and policymakers, local NGO's working in ECD and child-care centres are expected to facilitate fast-cycle learning, promote buy-in, dissemination of study findings and ultimately promote sustainability of the programme. Results will be disseminated to the local community including various key stakeholders through dissemination meetings and workshops. The Policy Engagement and Communications Unit staff at APHRC will facilitate sharing of innovation information through appropriate media outlets including policy briefs, blogs and twitter and others. The results will be disseminated to the scientific community through publication in peer-reviewed journals and presentations at local and international conferences.

\section{Collaborators}

This study is led by co-principal investigators; Dr Helen Elsey (UoY) and Dr Margaret Nampijja (APHRC). Dr Elsey is a public health specialist and researcher on urban health. She brings expertise in public health intervention development and the use of mixed methods within implementation research. Dr Nampijja is a clinical developmental psychologist with research focus and long experience in ECD. She brings ECD expertise and medical input to the project. Mr Kenneth Okelo has a background in early childhood education, and long experience in management of mixed methods ECD projects within the urban-poor settings in Nairobi. Mr Okelo brings his research management and community engagement skills to the project. Mr Afzal Habib is the Director of Kidogo; he brings extensive experience of working with 'mamaprenuers' to help improve the quality of their child-care centres through a business model. Dr Patricia Kitsao-Wekulo is a developmental psychologist who has led several studies in ECD. Her expertise in evaluating the impact of the child's environment and interactions on developmental outcomes is important for the study. 
Dr Elizabeth Kimani-Murage is a public health nutrition specialist and she advises on the nutrition aspects of the child-care environment.

Contributors MN led the preparation of the manuscript with inputs from HE. All the authors contributed to the conceptualisation, design and writing of the protocol from which this manuscript was developed. MN, HE and KO developed the quantitative and qualitative data collection tools and methods. All authors (MN, $\mathrm{KO}$, PKW, EKM and HE) participated in the writing, reviewing and editing of the final version of the manuscript.

Funding This work is supported by the British Academy, grant number ECE190115. Competing interests None declared.

Patient and public involvement Patients and/or the public were not involved in the design, or conduct, or reporting, or dissemination plans of this research.

Patient consent for publication Not required.

Provenance and peer review Not commissioned; externally peer reviewed.

Open access This is an open access article distributed in accordance with the Creative Commons Attribution Non Commercial (CC BY-NC 4.0) license, which permits others to distribute, remix, adapt, build upon this work non-commercially, and license their derivative works on different terms, provided the original work is properly cited, appropriate credit is given, any changes made indicated, and the use is non-commercial. See: http://creativecommons.org/licenses/by-nc/4.0/.

ORCID iD

Margaret Nampijja http://orcid.org/0000-0002-1200-6042

\section{REFERENCES}

1 Black MM, Walker SP, Fernald LCH, et al. Early childhood development coming of age: science through the life course. Lancet 2017;389:77-90.

2 Britto PR, Lye SJ, Proulx K, et al. Nurturing care: promoting early childhood development. Lancet 2017;389:91-102.

$3 \mathrm{CSDH}$. Closing the gap in a generation Health equity through action on the social determinants of health Commission on Social Determinants of Health FINAL REPORT CLOSING THE GAP IN A GENERATION CONTENTS [Internet], 2008. Available: https://apps. who.int/iris/bitstream/handle/10665/43943/9789241563703_eng. pdf?sequence=1 [Accessed cited 2020 Jun 15].

4 Richter LM, Daelmans B, Lombardi J, et al. Investing in the foundation of sustainable development: pathways to scale up for early childhood development. Lancet 2017;389:103-18.

5 Shonkoff JP, Garner AS, et al, Committee on Psychosocial Aspects of Child and Family Health. The lifelong effects of early childhood adversity and toxic stress. Pediatrics 2012;129:e232-46.

6 United Nations. Transforming our world: the 2030 agenda for sustainable development United nations United nations transforming our world: the 2030 agenda for sustainable development. AV RES/70/1. United Nations 2015.

7 WHO/UNICEF/WB. nurturing care for early childhood development: a framework for helping children survive and thrive to transform health and human potentia. Medico e Bambino 2018.

8 Meuwissen AS, Carlson SM. Fathers matter: the role of father parenting in preschoolers' executive function development. J Exp Child Psychol 2015;140:1-15.

9 United Nations. World urbanization prospects 2018. Webpage, 2018

10 Vlahov D, Freudenberg N, Proietti F, et al. Urban as a determinant of health. J Urban Heal 2007

11 Calderón G. The effects of child care provision in Mexico. Banco México Work Pap, 2014.

12 Dang $\mathrm{H}-\mathrm{AH}$, Hiraga M. Nguyen CV. Childcare and Maternal Employment: Evidence from Vietnam, GLO Discussion Paper, No. 349, Global Labor Organization (GLO) [Internet], 2019. Available: http://hdl.handle.net/10419/196844www.econstor.eu [Accessed cited 2020 Jun 15]

13 Esping-Andersen G. After the golden age? welfare state dilemmas in a global economy. In: The Global Social Policy Reader, 2009.

14 Gelbach JB. Public schooling for young children and maternal labor supply. Am Econ Rev 2002.
15 Sanfelice V. Universal Public Childcare and Labor Force Participation of Mothers in Brazil [Internet], 2019. Available: https://drive.google. com/file/d/1QHhkDaGfFLcyGVZ9-1tjICpMIEe1cqO2/view [Accessed cited 2020 Jun 15]

16 Leroy JL, Gadsden P, Guijarro M. The impact of daycare programmes on child health, nutrition and development in developing countries: a systematic review. J Dev Eff 2012.

17 van Urk FC, Brown TW, Waller R, et al. Centre-Based day care for children younger than five years of age in high-income countries. Cochrane Database Syst Rev 2014:CD010544.

18 Nampijja M, Kizindo R, Apule B, et al. The role of the home environment in neurocognitive development of children living in extreme poverty and with frequent illnesses: a cross-sectional study. Wellcome Open Res 2018;3:152.

19 Brown TW, van Urk FC, Waller R, et al. Centre-Based day care for children younger than five years of age in low- and middle-income countries. Cochrane Database Syst Rev 2014:CD010543.

20 Hodges EA, Smith C, Tidwell S, et al. Promoting physical activity in preschoolers to prevent obesity: a review of the literature. J Pediatr Nurs 2013;28:3-19.

21 Vanderloo LM, Tucker P, Johnson AM, et al. The influence of centrebased childcare on preschoolers' physical activity levels: a crosssectional study. Int J Environ Res Public Health 2014;11:1794-802.

22 Vohra R, Madhavan S, Sambamoorthi U, et al. Access to services, quality of care, and family impact for children with autism, other developmental disabilities, and other mental health conditions. Autism 2014;18:815-26.

23 Grantham-McGregor S, Cheung YB, Cueto S, et al. Developmental potential in the first 5 years for children in developing countries. Lancet 2007;369:60-70.

24 Republic of Kenya. Early Childhood Development Service Standard Guidelines [Internet], 2006. Available: https://planipolis.iiep.unesco. org/sites/planipolis/files/ressources/kenya_ecd_service_standard_ guidelines_final.pdf [Accessed cited 2020 Jun 15].

25 Caddy E. There are at least 2700 informal child daycares in Nairobi Tiny Totos is working to help them upgrade their services [Internet]. Theirworld, 2017. Available: https://theirworld.org/voices/tinytotos-kenya-helping-informal-daycares-in-nairobi-upgrade-services [Accessed cited 2020 Jun 16]

26 Teddlie C, Tashakkori A. Foundations of mixed methods research: integrating Qualtitative and qualitative approaches in the Socia and behavioural sciences. SAGE Publications, Inc, 2009.

27 Beguy D, Elung'ata P, Mberu B, et al. Health \& Demographic Surveillance System Profile: The Nairobi Urban Health and Demographic Surveillance System (NUHDSS). Int J Epidemiol 2015;44:462-71.

28 Harms T, Cryer D, Clifford RM. Family Child Care Environment Rating Scale, Revised Edition (FCCERS-R) [Internet]. New York, NY: Teachers College Press, 2007. Available: https://fpg.unc.edu/node/ 1954 [Accessed cited 2020 Jun 16].

29 Point of Care Foundation. Experience Based Co-Design Toolkit co-design toolkit [Internet], 2011. Available: https://www.pointofc arefoundation.org.uk/resource/experience-based-co-design-ebcdtoolkit/ [Accessed cited 2020 Feb 5].

30 McDonald J. Communities of Practice. In: International Encyclopedia of the Social \& Behavioral Sciences. Second Edition, 2015.

31 Bridge. Early Childhood Development Communities of Practice [Internet], 2019. Available: https://www.bridge.org.za/get-involved/ communities-of-practice/early-childhood-development-communitiespractice/ [Accessed cited 2020 Jun 16]

32 Serrat O. Learning Histories [Internet]. Washington, DC: Asian Development Bank, 2010. Available: https://core.ac.uk/download/ pdf/5132797.pdf [Accessed cited 2020 Feb 2].

33 Gale NK, Heath G, Cameron E, et al. Using the framework method for the analysis of qualitative data in multi-disciplinary health research. BMC Med Res Methodol 2013;13:117.

34 Buse K, Mays N, Walt G. Making health policy (understanding public health). UK Bell Brain Ltd, 2005.

35 UN Women. Progress of the World's Women 2015-2016 [Internet], 2015. Available: https://www.unwomen.org/en/news/in-focus/csw61/ women-in-informal-economy\#notes [Accessed cited 2020 Jun 15].

36 Samman E, Presler-Marshall E, Jones N. Women's work mothers children and the global childcare crisis [Internet], 2016. Available: https://www.odi.org/sites/odi.org.uk/files/odi-assets/publicationsopinion-files/10333.pdf [Accessed 15 Jun 2020].

37 McFarlane C. Rethinking Informality: politics, crisis, and the City. Plan Theory Pract 2012. 